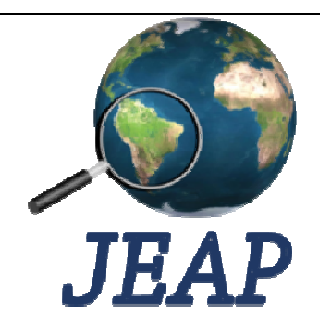

ISSN: 2525-815X

\section{Journal of Environmental} Analysis and Progress

Journal homepage: www.ufrpe.br/jeap http://dx.doi.org/10.24221/jeap.2.1.2017.1033.44-49

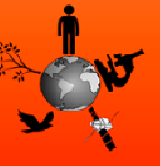

\title{
Biossorção do corante índigo carmim por Pennisetum purpureum Schumach. 1827 (Poales: Poaceae) (Capim elefante)
}

\section{Biosorption of the dye indigo carmine by Pennisetum purpureum Schumach. 1827 (Poales: Poaceae) (Elephant grass)}

\begin{abstract}
Aline Carla Costa do Nascimento ${ }^{\mathrm{a}}$, Iranildo José da Cruz Filho ${ }^{\mathrm{a}}$, Valmir Felix de Lima ${ }^{\mathrm{a}}$, Agrinaldo Jacinto do Nascimento Junior ${ }^{\mathrm{b}}$, Olga Martins Marques ${ }^{\mathrm{a}}$, Manuella Virginia Salgueiro Gondim ${ }^{\mathrm{a}}$

${ }^{a}$ Universidade Federal de Pernambuco-UFPE. Campus Recife. Av. Professor Moraes Rego, 1235. Cidade Universitária. Recife, Pernambuco. Brasil. CEP: 50670-901. E-mail: enilacarla23@gmail.com, iranildoj@gmail.com, olgammarques@gmail.com, atrazina@gmail.com, manuellavirginia@yahoo.com.br.

${ }^{\mathrm{b}}$ Instituto Federal de Educação Ciência e Tecnologia do Paraná. Campus Paranavaí. Rua José Felipe Tequinha, nº 1400. Jardim das Nações. Paranavaí, Paraná. Brasil. CEP: 87703-536. E-mail: agrinaldo18@ gmail.com.
\end{abstract}

\section{A R T I C L E I N F O}

Recebido 04 Nov 2016

Aceito 11 Jan 2017

Publicado 31 Jan 2017

\begin{abstract}
A B S T R A C T
Textile industries must treat their wastewater before discharge into hydric bodies. This treatment aims at removing the majority of colorants, whose interaction with the environment can cause great damage to nature. The biosorption has often shown an economical and effective technique in removing many of these dyes. Thus is proposed to apply as biosorbent, the Pennisetum purpureum Schumach, 1827 (Poales: Poaceae). (Elephant grass), to treat wastewater containing the dye índigo carmine. The assays of dye removal were conducted in batch mode under the following conditions: varying temperatures $\left(30,40,60\right.$ and $\left.100 \pm 2^{\circ} \mathrm{C}\right)$; constant agitation of $150 \mathrm{rpm} ; \mathrm{pH} 5.5 ; 0.5 \mathrm{~g}$ of biosorbent; and different concentrations of dye, 25 to $65 \mathrm{mg} . \mathrm{L}^{-1}$. The biosorption mechanism was well described by the Langmuir model. The maximum biosorption capacity was $17.51 \mathrm{mg} . \mathrm{g}^{-1}$ of elephant grass dry mass. Thermodynamics investigation indicated that favorable and spontaneous process at temperatures up to $60^{\circ} \mathrm{C}$, above that, the system showed a reduction in adsorption capacity. Thus, the use of $P$. purpureum Schumach, 1827 (Poales: Poaceae) as biosorbent for the removal of indigo carmine dye textile effluents showed as a new effective and economical alternative.
\end{abstract}

Keywords: Biosorption, indigo carmine, elephant grass.

\section{R E S U M O}

As indústrias têxteis devem tratar seus efluentes antes do descarte em corpos hídricos. Esse tratamento objetiva majoritariamente a remoção de corantes, cuja interação com o meio ambiente pode causar grandes danos a natureza. A Biossorção tem se mostrado muitas vezes uma técnica econômica e eficaz na remoção de boa parte destes corantes. Deste modo propomos aplicar como biossorvente, o Pennisetum purpureum Schumach, 1827 (Poales: Poaceae) (Capim elefante), para tratar efluentes contendo o corante índigo carmim. Os ensaios de remoção do corante foram realizados nas seguintes condições: variando as temperaturas $\left(30,40,60\right.$ e $\left.100 \pm 2^{\circ} \mathrm{C}\right)$, com agitação constante de $150 \mathrm{rpm}, \mathrm{pH}$ de 5,5 , utilizando $0,5 \mathrm{~g}$ de biossorvente, sob diferentes concentrações de corante (de 25 a $\left.65 \mathrm{mg} . \mathrm{L}^{-1}\right)$. O mecanismo de biossorção foi adequadamente descrito pelo modelo de Langmuir. A capacidade biossortiva máxima foi de $17,51 \mathrm{mg} \cdot \mathrm{g}^{-1} \mathrm{de}$ matéria seca de capim elefante. A investigação termodinâmica indicou que a biossorção é um processo favorável e espontâneo em temperaturas até $60^{\circ} \mathrm{C}$, acima 
disso o sistema apresentou uma redução da capacidade biossortiva. A utilização de $P$. purpureum Schumach., 1827 (Poales: Poaceae) como biossorvente para a remoção do corante índigo carmim de efluentes têxteis se mostrou uma nova alternativa eficaz e econômica.

Palavras-Chave: Biosorção, índigo carmim, capim elefante.

\section{Introdução}

A preservação do meio ambiente é um tema de grande interesse para a sociedade, nesse contexto é notável a busca por processos químicos cada vez mais verdes e que gerem menos resíduos tóxicos. Dentre as tecnologias mais estudadas para remediar efluentes contaminados por corantes ou metais pesados está a biossorção. A técnica baseia-se na captação passiva de um poluente presente no efluente através da superfície de um material de origem biológica (Akso, 2005). O processo envolve uma fase sólida e outra fase líquida contendo uma ou mais espécies dissolvidas aptas a biossorção (poluentes), essa remoção pode ocorre por diferentes mecanismos, interações eletrostáticas, troca iônica, complexação, quelação e microprecipitação (Akso, 2005). As principais vantagens em se utilizar o método são: baixo custo do biossorvente; ausência de produtos secundários tóxicos durante o tratamento; reuso do biossorvente; regeneção do poluente sem perda das suas características físicas e químicas; alta eficiência; e curto tempo de processo (Crini, 2005).

O Brasil se encontra em uma posição privilegiada em relação ao aproveitamento integral das biomassas, isso porque o país apresenta grandes extensões de culturas agrícolas, vasta biodiversidade, água em abundância, intensa radiação solar e diversidade climática. Por ano, no país, são produzidos, em média, 350 milhões de toneladas de resíduos lignocelulósicos (Pereira et al., 2008). Uma planta com biomassa bastante promissora na remoção de poluentes em água é a espécie Pennisetum purpureum Shumach 1827 (Poales: Poaceae) (Capim - elefante), por apresenta alta produtividade e ser de fácil obtenção. Materiais de origem biológica se tornam bastante atrativos para o processo biossortivo, pois estes possuem diversos grupos funcionais, tais como carboxilas, hidroxilas, sulfatos, fosfatos e grupos amina, capazes remover contaminantes (Cardoso et al., 2011).

A capacidade de remoção de um poluente por um biossorvente é controlada pelo equilíbrio de fase. Para sistemas simples é possível construir uma curva de concentração do soluto na fase sólida, em função da concentração da fase fluída. Essas curvas dependentes da temperatura são denominadas de Isotermas de biossorção, as quais podem descrever como os poluentes interagem na superfície do biossorvente (Aksu, 2005). Estas isotermas ainda podem auxiliar na determinação da razão máxima de remoção de poluente pela área de cada tipo de biossorvente em condições específicas para se obter informações sobre os mecanismos de remoção (Dotto et al., 2011).

Um grande número de modelos tem sido empregado, para explicar o processo de remoção de poluentes por biossorventes, sendo os mais comumente citados na literatura aqueles propostos por Langmuir e Freundlich (Dotto et al., 2011; Barka et al., 2011). O modelo de Langmuir assume uma superfície do biossorvente com sítios de biossorção uniformes e a formação de uma monocamada de contaminante. $\mathrm{O}$ modelo de Freundlich considera a biossorção ocorrendo em multicamadas, se mostrando ser bastante útil para descrever superfícies altamente heterogêneas. Os parâmetros termodinâmicos são estudados visando obter informações sobre o processo de biossorção do corante (Barka et al., 2011).

Neste sentido, este estudo objetiva apresentar um processo eficiente para a remoção do corante índigo carmim pelo processo de biossorção, utilizando uma gramínea como biossorvente, a Pennisetum purpureum Schumach 1827 (Poales: Poaceae) (Capim elefante).

\section{Material e Métodos}

Preparo do material biossorvente e da solução de corante

Amostras de Pennisetum purpureum Schumach. 1827 (Poales: Poaceae) (Capim elefante) foram coletadas em um canteiro no Departamento de Zootecnia, da Universidade Federal Rural de Pernambuco (UFRPE). O capim foi cortado, lavado com água destilada e seco a $75^{\circ} \mathrm{C}$, por $72 \mathrm{~h}$. Despois de seco foi triturado em moinho de facas, tamisado em peneiras de Tyler $(1,43 \mathrm{~mm})$. Em seguida, foi submetido a um tratamento químico com $\mathrm{NaOH}$ 0,1 mol.L ${ }^{-1}$ durante $1 \mathrm{~h}$ (Chaves et al., 2008). Após o tratamento, as frações foram lavadas com água destilada, filtradas e secas a $75^{\circ} \mathrm{C}$ por $72 \mathrm{~h}$.

O corante sintético utilizado foi o índigo carmim (Figura 1) da Sigma-Aldrich. No preparo das soluções utilizou-se água destilada. As concentrações das soluções foram diluídas a partir de uma solução estoque. Para o ajuste do pH das soluções utilizou-se ácido clorídrico e hidróxido de sódio. A determinação da concentração do corante realizou-se por espectroscopia no 
ultravioleta visível (UV/Vis), no comprimento de onda de $610 \mathrm{~nm}$.<smiles>CS(=O)(=O)c1ccc2c(c1)C(=O)/C(=C1/Nc3ccc(S(C)(=O)=O)cc3C1=O)N2</smiles>

Figura 1. Estrutura molecular do corante índigo carmim.

\section{Influência do $\mathrm{pH}$ no processo biossortivo}

O biossorvente foi submetido a ensaios prévios, conduzidos em sistema de batelada, em diferentes pHs (2 a 9) nas seguintes condições: concentração de corante $25 \mathrm{mg} . \mathrm{L}^{-1}, 150 \mathrm{rpm}$ a $30^{\circ} \mathrm{C}$ por $12 \mathrm{~h}$ e $0,5 \mathrm{~g}$ do Capim elefante seco, objetivando encontrar a condição de melhor adsorção do sistema calculada pela equação 1 .

$$
q=\frac{\left(c_{0}-c_{e}\right)}{M} V
$$

onde $\mathrm{C}_{0}$ é concentração do soluto na solução inicial $\left(\mathrm{mg} . \mathrm{L}^{-1}\right) ; \mathrm{C}_{\mathrm{e}}$, concentração residual no equilíbrio (mg.. $\left.\mathrm{L}^{-1}\right)$; V, volume da solução $(\mathrm{L}) ; \mathrm{m}$, massa do adsorvente $(\mathrm{g})$.

Estudo de equilíbrio e parâmetros termodinâmicos de biossorção

Os ensaios de equilíbrio foram realizados em triplicata, nas seguintes condições: $0,5 \mathrm{~g}$ de

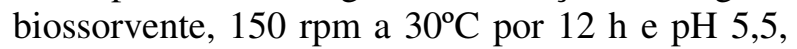
variando apenas as concentrações da solução de corante $25,35,45,55$ e $65 \mathrm{mg} . \mathrm{L}^{-1}$, por $2 \mathrm{~h}$. Os modelos utilizados foram os de Langmuir e de Freundlich de acordo com as Equações 2 e 3, respectivamente:

$$
\frac{C_{\theta}}{q_{\theta}}=\frac{1}{K_{L}}+\frac{a_{L}}{K_{L}} C_{\text {g }}
$$

$$
\ln q_{\theta}=\ln K_{f}+\frac{1}{n} C_{\theta}
$$

onde: $\mathrm{q}_{\mathrm{e}}$ a capacidade biossortiva no ponto de saturação em mg. $\mathrm{g}^{-1}, \mathrm{C}_{\mathrm{e}}$ é a concentração residual no equilíbrio, $\mathrm{K}_{\mathrm{L}}$ e $\mathrm{a}_{\mathrm{L}}$ representam as constantes de Langmuir e a capacidade e energia de biossorção e $K_{f}$ a constante de Freundlich.
A quantificação da concentração do corante em cada etapa do processo de remoção foi avaliada através da leitura da absorbância a 610 $\mathrm{nm}$ (Chaves et al., 2008) e convertida em mg.L $\mathrm{L}^{-1}$, através de uma curva analítica pré-estabelecida.

As características termodinâmicas do processo foram expressas em diferentes temperaturas e concentrações, através das variações de entropia, entalpia, energia livre de Gibbs e energia de ativação, pelas Equações 4, 5 e 6 , respectivamente:

$$
\begin{aligned}
& \ln K=\frac{\Delta H^{\circ}}{R T}+\frac{\Delta S^{\circ}}{R} \\
& \Delta G^{\circ}=-R T \ln K
\end{aligned}
$$

$$
\ln K=\ln A+\frac{E_{a}}{R T}
$$

onde: $\mathrm{K}$ é a constante de equilíbrio termodinâmico $\left(\mathrm{L} \cdot \mathrm{g}^{-1}\right), \Delta \mathrm{H}^{\circ}, \Delta \mathrm{S}^{\circ}$ e $\Delta \mathrm{G}^{\circ}$ entalpia, entropia e energia livre de Gibbs do sistema. $\mathrm{R}$ é a constante universal dos gases $\left(8,314 \mathrm{~J} \cdot \mathrm{mol}^{-1} \cdot \mathrm{K}^{-1}\right)$, T é a temperatura $(\mathrm{K})$. $\mathrm{E}_{\mathrm{a}}$ Energia de ativação e $\mathrm{A}$ é o fator de frequência de Arrhenius.

\section{Resultados e Discussão}

Influência do $\mathrm{pH}$

A Figura 2 mostra que o $\mathrm{pH}$ afeta, diretamente, a carga na superfície dos biossorventes, assim como influencia na ionização de diversos solutos. Este resultado pode estar relacionado com as interações intermoleculares não covalentes que ocorrem entre o corante e o biosorvente. Com a diminuição do $\mathrm{pH}$ do sistema, a quantidade de sítios carregados positivamente aumentam, enquanto os sítios carregados negativamente diminuem. Segundo Al-Ghouti et al. (2003), a capacidade de biossorção de corantes aniônicos é favorecida pela diminuição do $\mathrm{pH}$.

\section{Isotermas e parâmetros termodinâmicos de biossorção}

Através dos ensaios de equilíbrio foram obtidas as isotermas de biossorção nas temperaturas estudadas. Os pontos experimentais foram ajustados linearmente aos modelos de Langmuir e Freundlich (Figura 3) a fim de descrever o equilíbrio termodinâmico. De acordo com a linearização das equações de cada modelo e 
com os dados de cada gráfico, observa-se, a partir do coeficiente de determinação $\mathrm{R}^{2}$, que os resultados para a linearização ao modelo de Freundlich não foram satisfatórios, apenas para o modelo de Langmuir os resultados obtidos mostraram-se mais adequados. Os parâmetros calculados também evidenciam que $60^{\circ} \mathrm{C}$ é a temperatura que melhor se ajusta ao modelo; apresentando $\mathrm{R}^{2}=0,998$ superior as demais. $\mathrm{O}$ modelo de Langmuir considera que a biossorção ocorre em monocamadas, assume energias biossorção idênticas para cada molécula de contaminante e descreve a superfície do biossorvente homogênea.

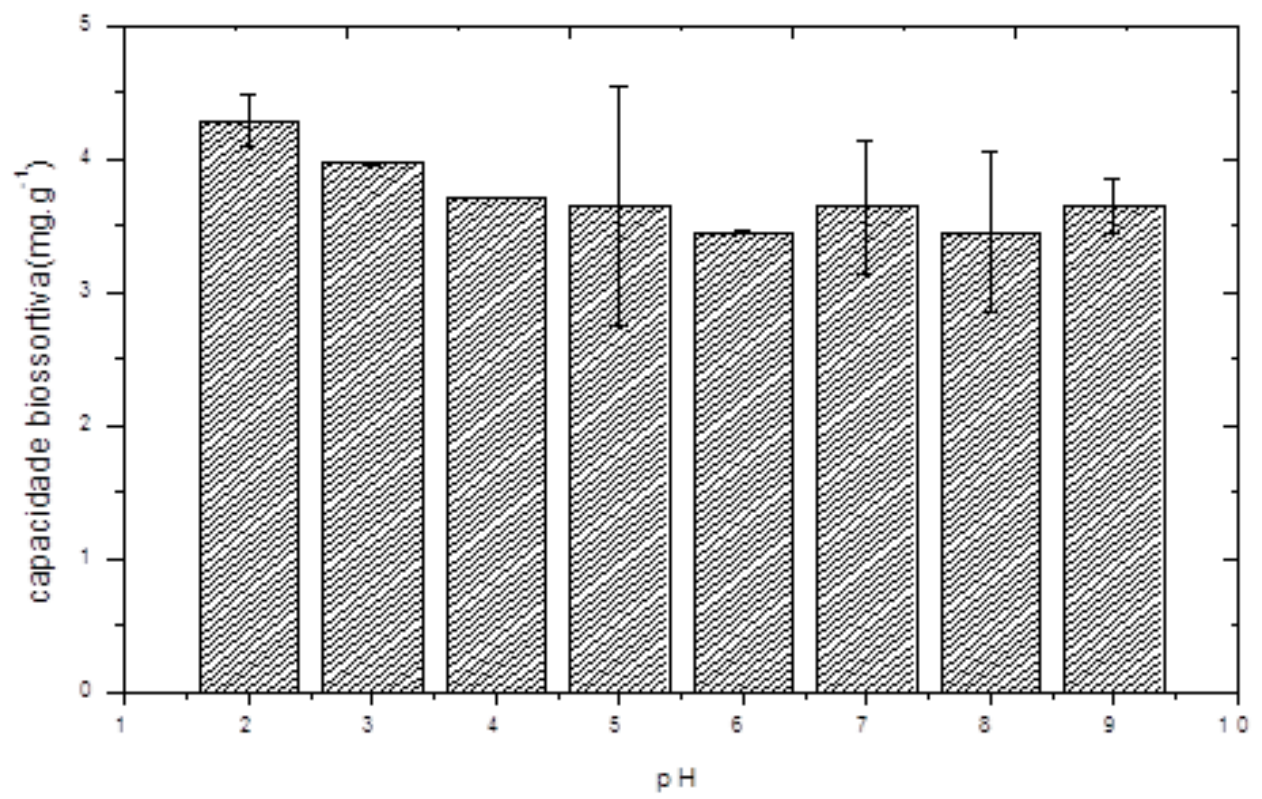

Figura 2. Influência do pH na remoção do corante índigo carmim.
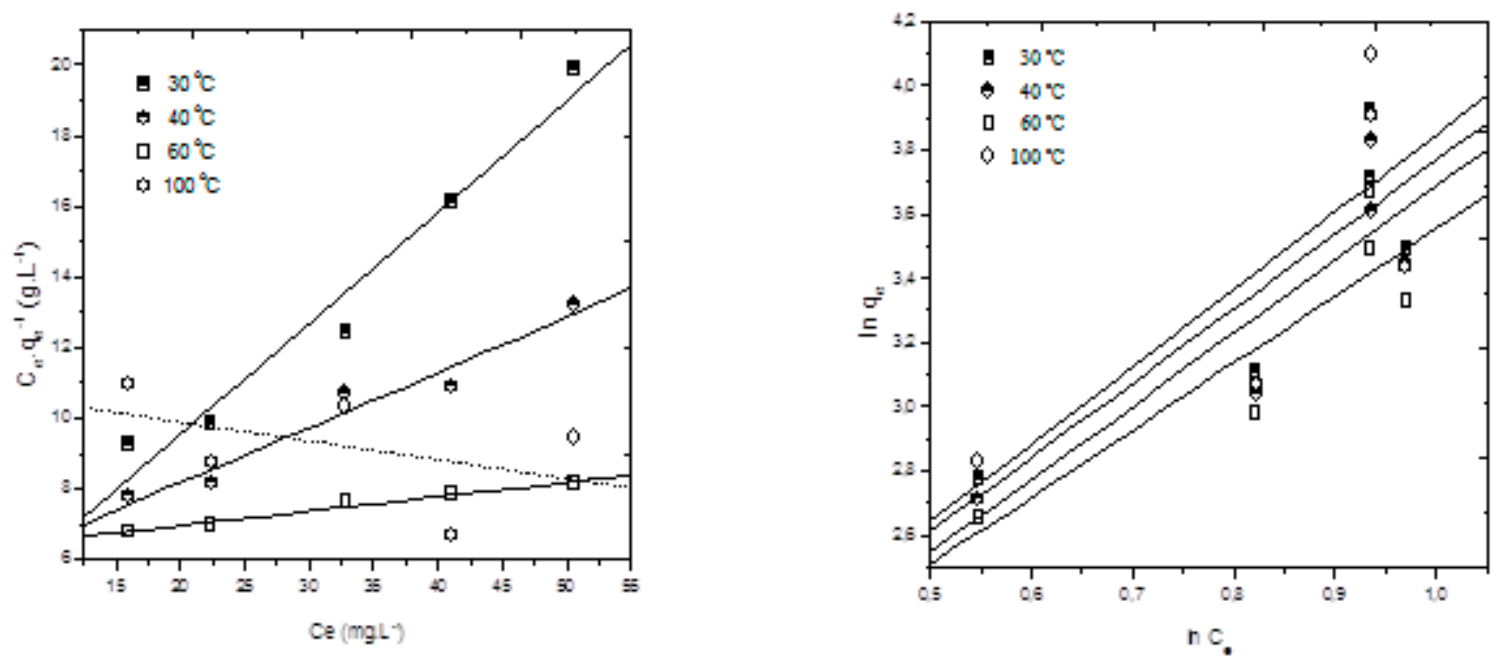

Figura 3. Ajuste dos dados de biossorção aos modelos de Langmuir e Freundlich a $30,40,60$ e $100^{\circ} \mathrm{C}$, respectivamente.

Observando os parâmetros obtidos na Tabela 1 verifica-se uma diminuição do $\mathrm{K}_{\mathrm{L}}$ com aumento da temperatura evidencia um processo de natureza exotérmica (Dotto et al., 2011; Barka et al., 2011). $\mathrm{O} \mathrm{K}_{\mathrm{L}}$ se refere à capacidade de biossorção em monocamada, com isso o aumento da temperatura até $60^{\circ} \mathrm{C}$ favoreceu o processo que apresentou remoção máxima de máxima de $\mathrm{Q}_{\max }=$ $17,51 \mathrm{mg} \cdot \mathrm{g}^{-1}$.

Acima de $60^{\circ} \mathrm{C}$ ocorre uma diminuição significativa da capacidade biossortiva, verificada pelos parâmetros determinados a $100^{\circ} \mathrm{C}$. Aksu \& Tezer (2005) utilizaram algas verdes da espécie Chlorella vulgaris Kessler \& Huss. 1992 (Chlorococcales: Oocystaceae) como biosorventes para a remoção de corantes têxteis em temperaturas que variavam de 25 a $55^{\circ} \mathrm{C}$. Aravindhan et al. (2007) utilizaram como biossorvente algas da espécie Caulerpa scalpelliformis (R. Brown ex Turner) e C. Agardh. 1820 (Caulerpales: Caulerpaceae) para remover o corante amarelo ouro básico em temperaturas 
entre 20 a $60^{\circ} \mathrm{C}$. Nos dois estudos os autores verificaram que, em temperaturas acima de $50^{\circ} \mathrm{C}$, ocorreu baixa eficiência de remoção. Os autores relacionam essa diminuição na eficiência do processo a possíveis danos nos sítios de biossorção na superfície do biossorvente, devido ao aumento da temperatura e, consequentemente, a atividade superficial é diminuída.

Tabela 1. Parâmetros de equilíbrio para os modelos Langmuir e de Freundlich.

\begin{tabular}{ccccccc}
\hline \multicolumn{2}{c}{ Langmuir } & \multicolumn{3}{c}{ Freundlich } \\
\hline $\mathbf{T}\left(\mathbf{0}^{\mathbf{0}} \mathbf{C}\right)$ & $\mathbf{K}_{\mathbf{L}}$ & $\mathbf{Q}_{\max }$ & $\mathbf{R}^{\mathbf{2}}$ & $\mathbf{K}_{\mathbf{f}}$ & $\mathbf{n}$ & $\mathbf{R}^{\mathbf{2}}$ \\
& $\left(\mathbf{L . g} \mathbf{g}^{-1}\right)$ & $(\mathbf{m g . g}$ & & & \\
\hline 30 & 0,31 & 3,17 & 0,751 & 4,322 & 3,465 & 0,635 \\
40 & 0,21 & 5,63 & 0,968 & 4,055 & 2,092 & 0,942 \\
60 & 0,17 & 17,51 & 0,998 & 4,323 & 1,239 & 0,996 \\
100 & 0,03 & 0,49 & 0,767 & 3,468 & 0,412 & 0,779 \\
\hline
\end{tabular}

Para avaliar o efeito da temperatura no processo foram aplicados os parâmetros termodinâmicos. A Tabela 2 mostra que o $\Delta \mathrm{H}^{\circ}$ é negativo, indicando que este é um processo exotérmico para as diferentes temperaturas investigadas. $\mathrm{O}$ valor de $\Delta \mathrm{H}^{\circ}$ é menor que 40 $\mathrm{kJ} \cdot \mathrm{mol}^{-1} \mathrm{e}$ o valor obtido pela energia de ativação, apresentando características de fisissorção, ocorrem por ligações fracas de Van der Waals (Al-Degs et al., 2000). O aumento da temperatura ocasionou uma notável diminuição na remoção. Valores negativos de $\Delta S^{o}$ sugerem uma redução da randomicidade na interface sólido-solução durante a biossorção do índigo carmim pelo capim elefante (Barka et al., 2011).

Tabela 2. Parâmetros termodinâmicos. $\Delta \mathrm{H}^{\circ}, \Delta \mathrm{S}^{\circ}$ e $\Delta \mathrm{G}^{\circ}$ para $30,40,60$ e $100^{\circ} \mathrm{C}$.

\begin{tabular}{|c|c|c|c|c|}
\hline $\begin{array}{l}\text { Temperatura } \\
\left({ }^{\circ} \mathrm{C}\right)\end{array}$ & $\frac{\mathbf{K}_{\mathbf{L}}}{\left(\mathbf{L} . \mathbf{g}^{-1}\right)}$ & $\begin{array}{c}\Delta \mathbf{G}^{\mathbf{0}} \\
\left(\mathbf{J}^{\prime} \mathbf{m o l}^{-1}\right)\end{array}$ & $\frac{\Delta \mathbf{H}^{\mathbf{0}}}{\left(\mathbf{k J . m o l} \mathbf{m o l}^{-1}\right)}$ & $\begin{array}{c}\Delta S^{\mathbf{o}} \\
\left(\mathbf{1 0 ^ { - 3 }} \mathbf{K}^{-1} \cdot \mathbf{m o l}^{-1}\right)^{-1}\end{array}$ \\
\hline 30 & 0,31 & $+2,67$ & & \\
\hline 40 & 0,21 & $+3,79$ & $-31,14$ & +112 \\
\hline 60 & 0,17 & $+6,02$ & & \\
\hline 100 & 0,03 & $+10,49$ & & \\
\hline
\end{tabular}

Com o aumento das temperaturas verificase que ocorre um aumento da energia livre de Gibbs, mostrando que o processo de biossorção é mais favorável em temperaturas abaixo de $100^{\circ} \mathrm{C}$, tendo sua condição ideal em $60^{\circ} \mathrm{C}$. O processo biossortivo apresentou energia de ativação de $\mathrm{E}_{\mathrm{a}}=$ $1,8 \mathrm{~kJ}^{\mathrm{mol}}{ }^{-1}$ (Figura 4).

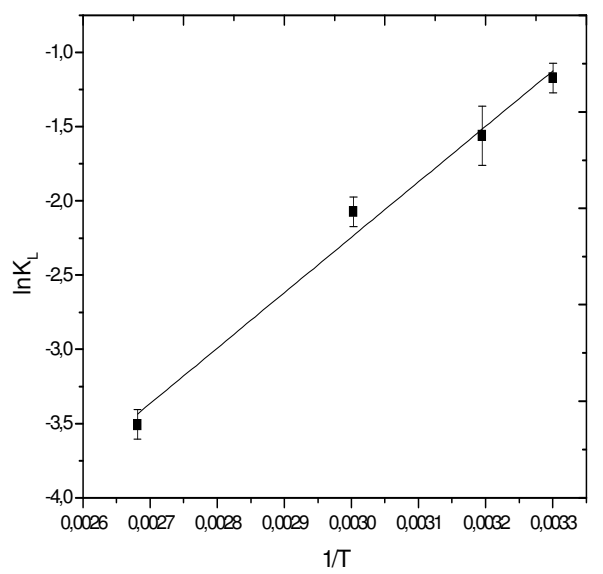

Figura 4. Gráfico de obtenção da energia de ativação do processo biossortivo.

\section{Conclusões}

O biossorvente capim elefante se mostrou como uma alternativa econômica e eficiente, enfatizada por um rendimento de $72 \%$ na remoção de cor de efluentes aquosos. A aplicação do modelo de Langmuir foi a mais adequada para a biossorção do corante índigo carmim. A melhor condição de biossorção foi em $60^{\circ} \mathrm{C}$ e temperaturas mais altas provocam uma diminuição da capacidade biossortiva do capim elefante.

A interação biossorvente-corante de natureza física agregam um maior valor ao biossorvente, ao possibilitar seu reaproveitamento após a remoção do corante.

\section{Referências}

AKSU, Z.; TEZER, S. 2005. Biosorption of reactive dyes on the green alga Chlorella vulgaris. Process Biochemistry, v.40, n.3/4, p.1347-1361.

AL-DEGS, Y.; KHRAISHEH, M. A. M.; ALLEN, S. J.; AHMAD, M. N. 2000. Effect of carbon surface chemistry on the removal of reactive dyes from textile effluent. Water Research, v.34, n.3, p.927-935. 
AL-GHOUTI, M. A.; KHRAISHEH, M. A. M.; ALLEN, S. J.; AHMAD, M. N. 2003. The removal of dyes from textile wastewater: a study of the physical characteristics and adsorption mechanisms of diatomaceous earth. Journal of Environmental Management, v.69, n.3, p.229-38.

ARAVINDHAN, R.; RAO, J. R.; NAIR, B. U. 2007. Removal of basic yellow dye from aqueous solution by sorption on green alga Caulerpa scalpelliformis. Journal of Hazardous Materials, v.142, n.1/2, p.68-76.

BARKA, N.; ABDENNOURI, M.; EL MAKHFOUK, M. 2011. Removal of Methylene Blue and Eriochrome Black $\mathrm{T}$ from aqueous solutions by biosorption on Scolymus hispanicus L. Kinetics, equilibrium and thermodynamics. Journal of the Taiwan Institute of Chemical Engineers, v.42, n.2, p.320-326.

CARDOSO, N. F.; LIMA, E. C.; PINTO, I. S.; AMAVISCA, C. V.; ROYER, B.; PINTO, R. B.; PEREIRA, S. F. 2011. Application of cupuassul sheel as biosorbent for the removal of textile dyes from aqueous solution. Journal Environmental Management, v.92, n.4, p.1237-1247.
CHAVES, K. O.; MONTEIRO, C. R. L.; MUNIZ, C. R.; GOMES, R. B.; BUARQUE, H. L. D. B. 2008. Adsorção de índigo carmim em biomassas mortas de Aspergillus niger. Engenharia sanitária e ambiental, v.13, n.4, p.351-355.

CRINI, G. 2005. Non-conventional low-cost adsorbents for dye removal: a review. Bioresource Technology, v.97, p.1061-1085.

DOTTO, G. L.; VIEIRA, M. L. G.; GONÇALVES, J. O.; PINTO, L. A. A. 2011. Remoção dos Corantes Azul brilhante, Amarelo Crepúsculo e Amarelo Tartrazina de Soluções Aquosas Utilizando Carvão Ativado, Terra Ativada, Terra Diatomácea, Quitina e Quitosana: Estudos de Equilíbrio e Termodinâmica. Química Nova, v.34, p.1193-1199.

PEREIRA, N.; COUTO, M. A. P. G.; SANTA A. L. M. M. 2008. Biomass of lignocellulosic composition for fuel ethanol production and the context of biorefinery. Series on Biotechnology, v.2, p.2-45. 